\title{
Effect of heat treatment on dynamic recrystallization behaviors and mechanical properties of $\mathrm{Mg}-2.7 \mathrm{Nd}-0.5 \mathrm{Zn}-0.8 \mathrm{Zr}$ alloy
}

Duxiu Zhang ${ }^{\text {a }}$, Xuyue Yang ${ }^{\text {a b, }}$, Zhengwu Fang ${ }^{\text {a }}$, Zhenyu Xiao ${ }^{\text {a }}$, Yi Yang ${ }^{\text {a }}$, Taku Sakai $^{\mathrm{c}}$

${ }^{a}$ Educational Key Laboratory of Nonferrous Metal Materials Science and Engineering, School of Materials Science and Engineering, Central South University, Changsha 410083, China

${ }^{\mathrm{b}}$ Nonferrous Metal Oriented Advanced Structural Materials and Manufacturing Cooperative Innovation Center, Changsha, 410083, China

${ }^{\mathrm{c}}$ UEC Tokyo (The University of Electro-Communications), Chofu, Tokyo 182-8585, Japan

* Corresponding author: Tel: +86-731-88876470, fax: +86-731-88830136

Email: yangxuyue@ mail.csu.edu.cn

Abstract The fine-grained structure development and the resultant mechanical properties during/after hot deformation were investigated in a $\mathrm{Mg}-2.7 \mathrm{Nd}-0.5 \mathrm{Zn}-0.8 \mathrm{Zr}$ alloy pretreated in solution and subsequently full aging. The true stress-true strain flow curves show a two-step work hardening during hot compression due to the interactions between dislocations and $\mathrm{Mg}-\mathrm{Nd}$ precipitates as well as $\mathrm{Zn}-\mathrm{Zr}$ intermetallics. In as-aging (AA) sample containing statically precipitated particles (SPPs), deformation bands (DBs) are easily developed at low strains and then ultrafine grains (UFGs) are evolved at along the boundaries of DBs and original grains at medium strains and rapidly approaches to a full development of final stable UFGs as the strain goes higher. In as-solution (AS) sample, by contrast, equiaxial UFGs are partially developed even in high strain and the grain sizes are smaller attributed to dynamic precipitated particles (DPPs). Tensile tests were carried out at ambient temperature for both types of sample, the results show that the tensile elongation to fracture of the hot deformed AA sample is higher than that of the AS one, because the former has a higher volume fraction and a weaker texture of UFGs developed. Besides, the AA sample contains a higher $5 \%$ proof strength compared 
with the AS one at low deformation temperature, however, such trend is reversed at high temperature, which may be resulted from the effect of SPPs and DPPs, respectively.

Keywords precipitates; hot compression; microstructure; ultrafine grain; microtexture; mechanical properties

\section{Introduction}

Magnesium $(\mathrm{Mg})$ alloys are the lightest available structural materials characterized by their low density, good castability, high specific strength and outstanding machinability, etc. Hence, they exert a tremendous fascination in many fields all over the world driven by the need of energy conservation, such as airplane, high-speed train, automobile and other industries [1-4]. Among various kinds of $\mathrm{Mg}$ alloys, $\mathrm{Mg}-\mathrm{Nd}-\mathrm{Zn}-\mathrm{Zr}$ alloys have drawn widespread attention because of their excellent comprehensive mechanical properties at ambient to elevated temperatures [5]. Specifically, Nd poses the highest solid solubility among all the rare earth (RE) elements, together with $\mathrm{Zn}$, can significantly improve the creep properties by the combination of precipitation and solid solution hardening [6-7]. Besides, it is reported that $\mathrm{Zr}$ element can efficiently promote grain refinement [1]. Recently, $\mathrm{Mg}-2.7 \mathrm{Nd}-0.5 \mathrm{Zn}-0.8 \mathrm{Zr}$ alloy, having moderate strength, good toughness and excellent corrosion resistance [8], has drawn more attention and been used as the shell components in the automotive industries as well as the aerospace field such as the engine crankcase and the oil pump cover [9]. These make the followed-up research on $\mathrm{Mg}-\mathrm{Nd}-\mathrm{Zn}-\mathrm{Zr}$ alloy become more and more necessary.

However, the industrial applications at ambient temperature of $\mathrm{Mg}$ alloys have been greatly limited due to their poor ductility resulted from the hexagonal close-packed (HCP) structure. Recent researches on $\mathrm{Mg}$ alloys have turned to study the improvement of formability caused by grain refinement taking place during thermo-mechanical processing [10,11, 12, 13]. Robson et al. [14] revealed that large

particles can promote the development of ultrafine grains (UFGs) by 
particle-stimulated nucleation mechanism in $\mathrm{Mg}-\mathrm{Mn}$ alloy. $\mathrm{Xu}$ et al. [15] found that the $\beta-\mathrm{Mg}_{17} \mathrm{Al}_{12}$ precipitated particles evolved in $\mathrm{AZ91} \mathrm{Mg}$ alloy can increase the volume fraction of UFGs having a rather weak basal texture. In a previous work, the authors have investigated the development behaviors of UFGs and the resultant mechanical properties of a $\mathrm{Mg}-8 \mathrm{Y}-2 \mathrm{Nd}-0.45 \mathrm{Zr}$ alloy pretreated in solution and subsequent aging followed by hot deformation. They found that the aging sample containing precipitated particles can approach a full development of UFGs and then achieve a higher tensile elongation at ambient temperature [16]. However, the $\mathrm{Mg}-\mathrm{Y}-\mathrm{Nd}-\mathrm{Zr}$ alloy is less suitable to be widely applied as $\mathrm{Y}$ addition is much more expensive than $\mathrm{Nd}$, compared to $\mathrm{Mg}-\mathrm{Nd}-\mathrm{Zn}-\mathrm{Zr}$ alloy. Although, some of the previous studies $[2,8,9]$ on $\mathrm{Mg}-\mathrm{Nd}-\mathrm{Zn}-\mathrm{Zr}$ alloy have investigated phase transformation taking place in age-hardening treatment [2] and the effect of precipitates on the microstructures and mechanical properties of as-cast sample. Any effect of precipitation particles on the formation of UFGs, however, has not been reported and discussed.

The present study was aimed to reveal the effect of pre-aging treatment on the development of UFGs during hot deformation of a $\mathrm{Mg}-2.7 \mathrm{Nd}-0.5 \mathrm{Zn}-0.8 \mathrm{Zr}$ alloy and the resultant mechanical properties at ambient temperature. Through a suitable thermo-mechanical process including aging treatment and high-speed hot compression, a high fraction of UFGs with some weakening of the basal texture was successfully developed, leading to the development of high ductility $\mathrm{Mg}$ alloy at ambient temperature. The dynamic formation process of UFGs in a $\mathrm{Mg}-2.7 \mathrm{Nd}-0.5 \mathrm{Zn}-0.8 \mathrm{Zr}$ alloy during hot compression was analyzed in detail and the mechanisms operated were discussed.

\section{Experimental}

The composition of the $\mathrm{Mg}$ alloy tested was $2.7 \% \mathrm{Nd}, 0.5 \% \mathrm{Zn}, 0.8 \% \mathrm{Zr}$ and balanced $\mathrm{Mg}$ (mass fraction). The alloy was prepared by $99.9 \% \mathrm{Mg}, 99.99 \% \mathrm{Zn}$, $\mathrm{Mg}-25 \% \mathrm{Nd}$ and $\mathrm{Mg}-30 \% \mathrm{Zr}$ master alloys by melting in an electric resistance 
furnace under a protective atmosphere of $\mathrm{CO}_{2}$ and $\mathrm{SF}_{6}$ in the ratio of 100:1. It was casted into cylindrical ingots with a diameter of $60 \mathrm{~mm}$ at a pouring temperature of $1013 \mathrm{~K}$ and a mould temperature of $473 \mathrm{~K}$. Rectangular samples with a dimension of $10 \mathrm{~mm} \times 8 \mathrm{~mm} \times 8 \mathrm{~mm}$ were machined from the as-casted cylindrical ingot and then solution treated at $808 \mathrm{~K}$ for $12 \mathrm{~h}$. A part of the samples was heated at a peak aging temperature of $473 \mathrm{~K}$ in an oil bath for $11 \mathrm{~h}$ after solution treatment. This is denoted here as the as-aged (AA) sample. The other one was treated only in solution at $808 \mathrm{~K}$ for $12 \mathrm{~h}$. This is called as the as-solution (AS) sample. Uniaxial compression tests were performed at the temperatures ranging from $683 \mathrm{~K}$ to $773 \mathrm{~K}$ under a strain rate of $0.3 \mathrm{~s}^{-1}$ using an Instron-type mechanical testing machine equipped with a water quenching apparatus. To minimize the friction between compression anvil and the $\mathrm{Mg}$ alloy samples, graphite powder was used as lubricant. Each sample was heated and kept for $5 \mathrm{~min}$ at corresponding deformation temperature.

The as-compressed samples were cut into halves along the compression axis. The microstructures evolved in the center were examined by optical microscopy (OM), scanning electron microscopy (SEM) and electron backscatter diffraction (EBSD) with orientation microscopy (OIM). Further detailed microstructures were investigated using transmission electron microscopy (TEM, JEM2000EX) operated at $200 \mathrm{kV}$. The TEM foils were prepared by ion milling method after mechanical grinding. In addition, the tensile tests were carried out at room temperature with an initial rate of $3 \times 10^{-3} \mathrm{~s}^{-1}$. Three specimens were used for each testing condition. The grain size was measured by conventional linear intercept method.

\section{Results and discussion}

\subsection{Flow stress behaviors}

Series of true stress-true strain compression flow curves for both the AA and AS samples, deformed at temperatures from $683 \mathrm{~K}$ to $773 \mathrm{~K}$, are presented in Fig. 1. The flow stress behaviors are clearly changed by deformation temperature as well as pre-heat treatment. And the flow stresses of the AA samples are always higher than 
those of the AS ones at each temperature. It is interesting to note in Fig. 1 that the shape of these flow curves is rather unique and shows difference with that in the previous results of a Mg-8Y-2Nd-0.45Zr alloy [16]. The latter one shows a rapid work hardening and a peak flow stress evolved at low to medium strains, followed by a flow softening and a steady-state flow in high strain. While those curves in Fig. 1 show a two-step work hardening flow during hot compression up to strains of $\varepsilon \cong 1.5$. Namely, the flow stress increases rapidly with early deformation and approaches a stress plateau with a gradual hardening flow up to strains of $\varepsilon \cong 0.5$. This may be called as a first-step work hardening flow. With further compression, the flow stress starts to increase again accompanying with a mild work hardening flow and approaches a peak flow stress at $\varepsilon \cong 1.5$. Compared with those AS ones, this second-step work hardening takes place more clearly in AA samples. The difference of flow stresses between both types of samples becomes more obvious with decreasing deformation temperature. Such unique deformation behaviors in the present $\mathrm{Mg}$ alloy may attribute to any changes of precipitation particles developed in aging treatment and subsequent hot deformation, as described in next sections 3.2 to 3.4 .

\subsection{Microstructures developed in the AA sample}

\subsubsection{Microstructures evolved before hot deformation}

Fig. 2 presents typical microstructures of the AA sample evolved at $773 \mathrm{~K}$ for $5 \mathrm{~min}$ before hot compression. It is observed in Fig. 2 (a) that many precipitates are formed in equiaxial grains with an average size of about $70 \mu \mathrm{m}$. Two kinds of precipitated particles are developed in the center of most grains and along grain boundaries, as indicated by ' $\mathrm{A}$ ' and ' $\mathrm{B}$ ' in Fig. 2 (a). The former microstructure observed by TEM is shown in Figs. 2 (b). It is apparent from Fig. 2 (b) that these precipitation particles are mainly composed of long rod-like shapes and may be suggested as $\mathrm{Zn}-\mathrm{Zr}$ intermetallic phases developed during solution treatment [17]. On the other hand, Fig. 2 (c) presents the SEM and TEM images of the precipitates along grain boundaries labelled 
' $\mathrm{B}$ ' in Fig. 2 (a) and the corresponding selected area diffraction pattern (SADP) is shown in Fig. 2(d). As depicted in Fig. 2 (c), many isolated precipitation particles distribute along grain boundaries in the AA sample and confirmed as the equilibrium $\mathrm{Mg}_{12} \mathrm{Nd} \beta$ phase which has a body-centered cubic (bcc) crystal structure according to the SADP result. These results are the same as those reported in $[9,18,19]$. In addition, Fig. 2 (e) depicts the high magnified TEM of the statically precipitated particles (SPPs) in grain interior. As it is evident, a uniform distribution of the precipitates is clearly visible in the matrix and the corresponding SADP is provided in Fig. 2 (f). The SPPs are identified to be the $\beta_{1}$ phase with orientation relationships with the Mg matrix phase. These precipitates have a face-centered cubic (fcc) crystal structure which is different from $\mathrm{Mg}_{12} \mathrm{Nd}$ phase.

Fig. 3 shows the TEM graph of the AA sample deformed at $773 \mathrm{~K}$ to $\varepsilon=0.2$. It can be clearly observed in Fig. 3 that many dislocations pile up around the $\mathrm{Mg}_{12} \mathrm{Nd}$ precipitates (Fig. 3 (a)) and the $\beta_{1}$ phase (Fig. 3 (b)). In other words, the SPPs play the role as obstacles to hinder the movement of dislocations. The existence of such high density precipitates in the present $\mathrm{Mg}$ alloy can effectively retard dislocation motion and also induce stress concentration at the grain boundaries [20].

\subsubsection{Microstructure changes during hot deformation}

Fig. 4 represents the optical microstructures of the $\mathrm{Mg}$ alloy deformed to a strain of 1.5 at various compression temperatures. As shown in Figs. 4 (a) to 4 (c), equiaxial original grains are pancaked by compression to $\varepsilon=1.5$ accompanying with the development of deformation bands (DBs) crossing retained grain interiors. The ultrafine grains (UFGs) formed along original grain boundaries and expand toward grain interiors with increase in deformation temperature. The average size and volume fraction of UFGs increase concurrently with rise in temperature as well. At the highest temperature of $773 \mathrm{~K}$ (Fig. 4 (d)), equiaxed UFGs are almost fully developed in a whole volume. It is well known [10, 21, 22, 23, 24] that DBs are frequently formed in the $\mathrm{Mg}$ alloys during high temperature compression owing to its intrinsic anisotropic 
plasticity. With temperature increasing, other slip systems become frequently operative, then any effect derived from the plasticity anisotropy gets weakened and contributes to the more homogeneous deformation. These can result in any increase of the average size and the volume fraction of UFGs.

Microstructure changes during hot deformation of the AA sample were observed in detail at $773 \mathrm{~K}$. The results are shown in Fig. 5. As can be seen from Fig. 5 (a) that few DBs and UFGs are introduced in coarse grain interiors after deformed to $\varepsilon=0.2$. Such microstructure contains high density $\mathrm{Mg}_{12} \mathrm{Nd}$ precipitates mostly located at near the grain boundaries. With further straining, more UFGs are developed at along the grain boundaries as well as deformation bands formed in grain interiors, and then UFGs developed at both the boundaries are combined with each other (Figs. 5 (b) and 5 (c)). Finally, these UFGs are almost fully evolved in a whole area at $\varepsilon=1.5$ (Fig. 5 (d)).

Fig. 6 shows some typical SEM images of the AA samples deformed to various strains at $773 \mathrm{~K}$. It is clearly seen in Fig. 6 that $\mathrm{Mg}_{12} \mathrm{Nd}$ precipitates are distributed at along both the boundaries of original grains and DBs introduced by early deformation. This phenomenon suggests that dynamic precipitation takes place during hot deformation to $\varepsilon=0.2$. In addition, long rod-like shaped $\mathrm{Zn}-\mathrm{Zr}$ intermetallics, as showed in the right-hand corner of Fig. 6 (a), are gradually changed to isolated finer ones at $\varepsilon=0.5$ (Fig. 6 (b)). With further deformed to $\varepsilon=1.5$, as depicted in Fig. 6 (c), all of these fine precipitates are distributed roughly perpendicular to the compression direction. It may be concluded from Fig. 6 that needle-like shaped Zn-Zr precipitates are broken and refined at low to medium strains and then redistributed during hot deformation as the strain goes higher.

\subsection{Microstructure changes during hot deformation of the AS sample}

Fig. 7 shows strain dependence of the microstructures developed in the AS sample tested at $773 \mathrm{~K}$. This is clearly in contrast with the microstructures of AA sample in Fig. 5. Namely, a few new grains and DBs are evolved at low strains and then grow 
gradually along the boundaries during early deformation. And the volume fraction of these UFGs developed in necklace is smaller than that in the AA sample. It is interesting to note in Fig. 7 (d) that UFGs partially develop even at high strains and the average size of UFGs is smaller than that evolved at lower strains. This may be resulted from the pinning effect led by dynamic precipitation taking place in the AS sample, which against grain growth and bring about the formation of finer UFGs.

Fig. 8 presents some typical SEM images of the AS samples deformed to various strains at $773 \mathrm{~K}$. It can be seen that long rod-like shaped $\mathrm{Zn}-\mathrm{Zr}$ intermetallics are rapidly broken into short rod-like shaped ones during the early deformation and then change to globular-like shaped ones after deformed to $\varepsilon=0.5$ (Figs. 8 (a) and 8 (b)). Further deformed to a high strain of $\varepsilon=1.5$, much finer precipitates are uniformly developed at the boundaries of newly developed UFGs with a size of around $5 \mu \mathrm{m}$ (Fig. 8 (c)). Fig. 9 shows the TEM image and corresponding SADP of the microstructure obtained from the AS sample deformed to $\varepsilon=1.5$ at $773 \mathrm{~K}$. As shown in Fig. 9, a lot of fine precipitates distributed at the boundary of UFGs. Meanwhile, these precipitate particles are identified as $\beta$ phase $\left(\mathrm{Mg}_{12} \mathrm{Nd}\right)$ according to the SADP result. This suggests that dynamic precipitation can concurrently take place accompanying with the formation of UFGs. Similar phenomenon was found in a previous hot compression experiment of a Mg-8Y-2Nd-0.45Zr alloy [16]. In addition, those $\beta$ phases can be hardly seen inside the original coarse grains and the rod-like shaped coarse Zn-Zr intermetallics are almost disappeared (Fig. 8 (d)).

It is well known in $\mathrm{Mg}$ alloys $[10,21,22]$ that UFGs are developed at along original grain boundaries at low strains and expand to grain interiors through the dynamic formation of kink bands, one kind of deformation bands (DBs)formed during hot deformation. Thus the formation of UFGs in Mg alloys can take place in-situ in segments fragmented by DBs and then be controlled by a series of strain-induced continuous reaction, i.e. continuous dynamic recrystallization (cDRX). In addition, UFGs may develop at low strains by the operation of necklace cDRX, as seen in Figs. 4, 5 and 7. This is, however, clearly distinguished from conventional necklace 
discontinuous DRX (dDRX), which takes place during hot deformation of face-centered cubic materials with low to medium stacking fault energy [21, 25]. The latter process contains two steps of nucleation and long-range growth, so the nature of dDRX is considered to be thermal. By contrast, necklace cDRX takes place through a single step strain-induced process therefore can be considered to be athermal [21].

\subsection{Quantitative analysis of the development of UFGs during hot deformation}

The volume fraction $\left(\mathrm{V}_{\mathrm{UFG}}\right)$ and grain size $\left(\mathrm{D}_{\mathrm{UFG}}\right)$ of UFGs developed by cDRX at $773 \mathrm{~K}$ were measured in the present $\mathrm{Mg}$ alloy by using the microstructures in Figs. 5 and 7. Changes in $\mathrm{V}_{\mathrm{UFG}}$ and $\mathrm{D}_{\mathrm{UFG}}$ with deformation are represented by solid line for the AA sample and by broken line for the AS one in Fig. 10. $V_{\mathrm{UFG}}$ for both types of samples increases gradually as strain rises to $\varepsilon \cong 0.5$, then it starts to increase rapidly at $\varepsilon>0.5$. At the highest strain of $\varepsilon=1.5, \mathrm{~V}_{\mathrm{UFG}}$ for the AA sample approaches roughly $100 \%$ and, by contrast, that for the AS one attains to only around $75 \%$. It is also remarkable to note in Fig. 10 (a) that $\mathrm{V}_{\mathrm{UFG}}$ for both kind of samples starts to increase gradually in low strain and then rapidly at high strains of $\varepsilon>0.5$. Such development behaviors of UFGs can be confirmed by the microstructural observations described in 3.2 and 3.3 .

Namely, Mg-Nd precipitates and Zn-Zr intermetallics may play an important role during the process. At early stage of deformation, $\mathrm{Mg}-\mathrm{Nd}$ precipitates and clusters composed of needle-like shaped $\mathrm{Zn}-\mathrm{Zr}$ intermetallics may effectively retard the development of DBs as well as UFGs. With further deformed to high strains, those particles are broken into finer ones and then the formation of UFGs may be easier to initiate and be accelerated into a whole volume. Moreover, such changes from coarse to finer phases may also result in the appearance of two-step work hardening flow of the present $\mathrm{Mg}$ alloy (see Fig. 1). That is to say, the first-step work hardening can take place through usual interactions between mobile dislocations and SPPs as well as the clusters of intermetallics existed in the present $\mathrm{Mg}$ alloy before deformation. The subsequent second-step work hardening flow may occur as a result of any interactions 
between dislocations and the finer particles.

On the other hand, as illustrated in Fig. 10 (b) that $\mathrm{D}_{\mathrm{UFG}}$ for the AA sample has a clear enhancement at strains from 0.2 to 0.9 , and then approaches a stable grain size of around $10 \mu \mathrm{m}$ at higher strains. $\mathrm{D}_{\mathrm{UFG}}$ for the AS sample, on the other hand, is roughly the same compared with that of the AA one at low strains, while it decreases inversely with further straining to $\varepsilon=1.5$, resulting in a much finer UFGs in high strain. The latter may be ascribed to a strong interaction between dynamic formation of UFGs and dynamic strain ageing, as confirmed in Figs. 8 and 9. Such effect, however, can hardly occur in the AA sample because of the full aging treatment before hot deformation. It is concluded, therefore, that statically precipitated particles (SPPs) preexisted in the AA sample can effectively retard for the initial formation of UFGs, but accelerate the formation of DBs at low strains (see Fig. 5 (a)), and subsequently promote the development of a final UFG structure in higher strain.

\subsection{Microtextures of UFG structures}

In order to discuss the mechanisms of UFG formation and texture development, the deformed microstructures of the present alloy were further investigated using EBSD. Typical OIM maps and their corresponding $\{0001\}$ pole figures, for both the AA and AS samples deformed to $\varepsilon=1.5$ at $773 \mathrm{~K}$, are represented in Fig. 11. Different colors indicate different crystallographic orientations, as defined in the inverse pole figure. It can be seen that equiaxial UFGs are developed almost fully in Fig. 11 (a), and partially in Fig. 11 (b) in a whole area. And the UFGs developed in the AA sample is coarser than that for the AS one in Fig. 11 (b), as confirmed also in Fig. 10 (b). Moreover, the maximum relative intensity of the texture for the AA sample is clearly lower than that for the AS one, i.e. 6.2 and 16.4.

It is also seen in Fig. 11 (b) that the basal planes of remained coarse grains rotate nearly perpendicular to the compression axis. The development of such (0001) basal plane texture is frequently reported in many $\mathrm{Mg}$ alloys deformed by compression [16, $22]$ and rolling $[26,27]$. It is concluded from Fig. 11 that a higher volume fraction of 
UFGs, evolved by cDRX, leads to the development of a weaker texture.

\subsection{Resultant mechanical properties after processing}

Engineering tensile stress-strain curves at ambient temperature for the present $\mathrm{Mg}$ alloy after thermo-mechanical processing are presented in Fig. 12. Both the AA and AS samples were hot deformed to $\varepsilon=1.5$, then rapidly quenched in water and finally machined to tensile specimens with a gauge size of $5 \mathrm{~mm} \times 1.5 \mathrm{~mm} \times 1.2 \mathrm{~mm}$. Some of the mechanical properties, e.g. total elongation to failure and $5 \%$ proof strength, are represented in Fig. 13. Elongation to fracture for both types of samples rises with increase in compression temperature and reaches different peaking values at $773 \mathrm{~K}$. Such value for the AA sample is always larger than that of the AS one because of a larger volume fraction of UFGs evolved in the former sample (Fig. 10). On the other hand, the $5 \%$ proof strength for the AA sample is higher than that of the AS one at low deformation temperature due to statically precipitated particles (SPPs). Nevertheless, the strength for the AS sample in high temperature is reversely higher than that for the AA one because the development of dynamically precipitated particles (DPPs). Then the difference of flow stresses in high strain for two kinds of samples decreases with increasing deformation temperature, as seen in Fig. 1.

\section{Conclusion}

Development behaviors of UFG formation during hot deformation of a $\mathrm{Mg}-2.7 \mathrm{Nd}-0.5 \mathrm{Zn}-0.8 \mathrm{Zr}$ alloy were studied in hot compression up to strain of 1.5 at temperatures from $683 \mathrm{~K}$ to $773 \mathrm{~K}$. The alloy samples were heat-treated in as-solution and subsequent aging and named as the AS and the AA samples, respectively. The main results obtained are summarized as follows:

(1) True stress-true strain flow curves of this alloy show a two-step work hardening during hot compression up to strains of 1.5. This can be attributed to the interactions between dislocations and $\mathrm{Mg}-\mathrm{Nd}$ precipitates as well as $\mathrm{Zn}-\mathrm{Zr}$ intermetallics. 
(2) In the AA sample containing statically precipitated particles (SPPs), deformation bands (DBs) can be easily developed at low strains and then UFGs are evolved at medium strains and rapidly approaches a full development of final stable UFGs as strain goes higher.

(3) In the AS sample, equiaxial UFGs are developed partially even in high strain and show a smaller size which resulted from dynamic precipitation taking place in the AS sample.

(4) Tensile elongation to fracture for the AA sample was $20 \%$, enhanced by $43 \%$ due to a higher volume fraction of UFGs with a weak texture developed by cDRX as compared to about $14 \%$ for the AS sample. In addition, the AA sample contains a higher $5 \%$ proof strength compared with AS one at low deformation temperature, and such trend is reversed at high temperature, which may be resulted from the effect of SPPs and DPPs, respectively.

\section{Acknowledgements}

The authors gratefully acknowledge the financial support received from the National Science Foundation of China (Grant No. 51474241) and the National Key Basic Research Program of China (Grant No. 2013CB632204).

\section{References}

[1] D. Orlov, D. Pelliccia, X. Fang, L. Bourgeois, N. Kirby, A.Y. Nikulin, K. Ameyama, Y. Estrin. Particle evolution in $\mathrm{Mg}-\mathrm{Zn}-\mathrm{Zr}$ alloy processed by integrated extrusion and equal channel angular pressing: Evaluation by electron microscopy and synchrotron small-angle X-ray scattering. Acta Mater. 72 (2014) $110-124$.

[2] A. Sanaty-Zadeh, A.A. Luo, D.S. Stone. Comprehensive study of phase transformation in age-hardening of $\mathrm{Mg}-3 \mathrm{Nd}-0.2 \mathrm{Zn}$ by means of scanning transmission electron microscopy. Acta Mater. 94 (2015) 294-306. 
[3] N. Stanford, M. Barnett. Effect of composition on the texture and deformation behaviour of wrought Mg alloys. Scr. Mater. 58 (2008) 179-182.

[4] J. Wang, D.X. Zhang, Y. Li, Z.Y. Xiao, J. P. Fouse, X.Y. Yang. Effect of initial orientation on the microstructure and mechanical properties of textured AZ31 Mg alloy during torsion and annealing. Mater. Des. 86 (2015) 526-535.

[5] F.Y. Zheng, Y.J. Wu, L.M. Peng, X.M. Li, P.H. Fu, W.J. Ding. Microstructures and mechanical properties of friction stir processed $\mathrm{Mg}-2.0 \mathrm{Nd}-0.3 \mathrm{Zn}-1.0 \mathrm{Zr}$ magnesium alloy. J. Magn. Alloy. 1 (2013) 122-127.

[6] P.A. Nuttall, T.J. Pike, B. Noble. Metallography of dilute Mg-Nd-Zn alloys. Metallography 13 (1980) 3-20.

[7] J.L. Yan, Y.S. Sun, F. Xue, S. Xue, W.J. Tao. Microstructure and mechanical properties in cast magnesium-neodymium binary alloys. Mater. Sci. Eng. A 476 (2008) 366-371.

[8] J.W. Chang, L.M. Peng, X.W. Guo, A. Atrens, P.H. Fu, W.J. Ding. Comparison of the corrosion behaviour in 5\% $\mathrm{NaCl}$ solution of $\mathrm{Mg}$ alloys $\mathrm{NZ30K}$ and AZ91D. J. Appl. Elect. 38 (2008) 207-214.

[9] P.H. Fu, L.M. Peng, H.Y. Jiang, J.W. Chang, C.Q. Zhai. Effects of heat treatments on the microstructures and mechanical properties of $\mathrm{Mg}-3 \mathrm{Nd}-0.2 \mathrm{Zn}-0.4 \mathrm{Zr}$ (wt.\%) alloy. Mater. Sci. Eng. A 486 (2008) 183-192.

[10] T. Sakai, H. Miura. Mechanical Properties of Fine-Grained Magnesium Alloys Processed by Severe Plastic Forging. Magnesium Alloys-Design, Processing and Properties, ed. F. Czerwinski, 2011, 219-244.

[11] J.J. Ma, X.Y. Yang, Q.H. Huo, H. Sun, J. Qin , J. Wang. Mechanical properties and grain growth kinetics in magnesium alloy after accumulative compression bonding. Mater Des 47 (2013) 505-509.

[12] Q. Chen, D.Y. Shu, Z.D. Zhao, Z.X. Zhao, Y.B. Wang, B.G. Yuan. Microstructure development and tensile mechanical properties of $\mathrm{Mg}-\mathrm{Zn}-\mathrm{Re}-\mathrm{Zr}$ magnesium alloy. Mater Des 40 (2012) 488-496.

[13] Q.M. Peng, X.L. Hou, L.D. Wang, Y.M. Wu, Z.Y. Cao, L.M. Wang. 
Microstructure and mechanical properties of high performance $\mathrm{Mg}$-Gd based alloys. Mater Des 30 (2009) 292-296.

[14] J.D. Robson, D.T. Henry, B. Davis. Particle effects on recrystallization in magnesium-manganese alloys: Particle-stimulated nucleation. Acta Mater. 57 (2009) 2739-2747.

[15] S.W. Xu, N. Matsumoto, S. Kamado, T. Honma, Y. Kojima. Effect of $\mathrm{Mg}_{17} \mathrm{Al}_{12}$ precipitates on the microstructural changes and mechanical properties of hot compressed AZ91 magnesium alloy. Mater. Sci. Eng. A 523 (2009) 47-52.

[16] D.X. Zhang, X.Y. Yang, H. Sun, Y. Li, J. Wang, Z.R. Zhang, Y.X. Ye, T. Sakai. Dynamic recrystallization behaviors and the resultant mechanical properties of a $\mathrm{Mg}-\mathrm{Y}-\mathrm{Nd}-\mathrm{Zr}$ alloy during hot compression after aging. Mater. Sci. Eng. A 640 (2015) 51-60.

[17] C.J. Bettles, M.A. Gibson, S.M. Zhu. Microstructure and mechanical behaviour of an elevated temperature Mg-rare earth based alloy. Mater. Sci. Eng. A 505 (2009) 6-12.

[18] J.L. Yan, Y.S. Sun, F. Xue, S. Xue, W.J. Tao. Microstructure and mechanical properties in cast magnesium-neodymium binary alloys. Mater. Sci. Eng. A 476 (2008) 366-371.

[19] Q. Li, Q.D. Wang, Y.X. Wang, X.Q. Zeng, W.J. Ding. Effect of Nd and Y addition on microstructure and mechanical properties of as-cast $\mathrm{Mg}-\mathrm{Zn}-\mathrm{Zr}$ alloy. J. Alloy. Compd. 427 (2007) 115-123.

[20] W.L. Xiao, S.S. Jia, J. Wang, J.L. Wang, L.M. Wang. Investigation on the microstructure and mechanical properties of a cast $\mathrm{Mg}-6 \mathrm{Zn}-5 \mathrm{Al}-4 \mathrm{RE}$ alloy. $\mathrm{J}$. Alloy. Compd. 458 (2008) 178-183.

[21] T. Sakai, A. Belyakov, R. Kaibyshev, H. Miura, J.J. Jonas. Dynamic and post-dynamic recrystallization under hot, cold and severe plastic deformation conditions. Prog. Mater. Sci. 60 (2014) 130-207.

[22] X.Y. Yang, H. Miura, T. Sakai. Dynamic Evolution of New Grains in Magnesium Alloy AZ31 during Hot Deformation. Mater. Trans. 44 (2003) 197-203. 
[23] Z.Y. Xiao, X.Y. Yang, Y. Yang, Z.R. Zhang, D. X. Zhang, Y. Li, T. Sakai. Microstructural development under interrupted hot deformation and the mechanical properties of a cast Mg-Gd-Y-Zr alloy. Mater. Sci. Eng. A 652 (2016) 377-383.

[24] X.Y. Yang, H. Miura, T. Sakai. Structural development at severely high strain in AZ31 magnesium alloy processed by cold forging and subsequent annealing. Mater Des 44 (2013) 573-579.

[25] T. Sakai, J.J. Jonas. Dynamic Recrystallization: Mechanical and Microstructural Considerations. Acta. Metal. 32 (1984) 189-209.

[26] J. Bohlen, M.R. Nurnberg, J.W. Senn, D. Lezig, S.R. Agnew. The texture and anisotropy of magnesium-zinc-rare earth alloy sheets. Acta Mater. 55 (2007) 2101-2112.

[27] W.J. Kim, J.B. Lee, W.Y. Kim, H.T. Jeong, H.G. Jeong. Microstructure and mechanical properties of $\mathrm{Mg}-\mathrm{Al}-\mathrm{Zn}$ alloy sheets severely deformed by asymmetrical rolling. Scr. Mater. 56 (2007) 309-312.

\section{Figure captions}

Fig. 1. Temperature dependence of true stress - true strain curves at $\dot{\varepsilon}=0.3 \mathrm{~s}^{-1}$ for $\mathrm{Mg}-\mathrm{Nd}-\mathrm{Zn}-\mathrm{Zr}$ alloy treated in as-solution (broken line) and subsequent aging (solid line).

Fig. 2. Microstructures of the AA sample heated at $773 \mathrm{~K}$ for $5 \mathrm{~min}$ before deformation. (a) optical micrograph, (b) TEM micrograph of some clusters of $\mathrm{Zn}-\mathrm{Zr}$ intermetallic phases marked 'A' in (a), (c) SEM and TEM and (d) corresponding SADP of the precipitates at grain boundary marked 'B' in (a), (e) TEM and (f) corresponding SADP of the precipitated particles in grain interiors.

Fig. 3. TEM image of the AA sample deformed to $\varepsilon=0.2$ at $773 \mathrm{~K}$.

Fig. 4. Microstructures of the AA sample deformed to a strain of 1.5 at various temperatures. (a) $683 \mathrm{~K}$, (b) $713 \mathrm{~K}$, (c) $743 \mathrm{~K}$ and (d) $773 \mathrm{~K}$. 
Fig. 5. Microstructures of the AA sample deformed to various strains at 773 K. (a) 0.2 , (b) 0.5 , (c) 0.9 and (d) 1.5 .

Fig. 6. Typical SEM images of the AA samples deformed to various strains at $773 \mathrm{~K}$. (a) $\varepsilon=0.2$, (b) $\varepsilon=0.5$, (c) $\varepsilon=1.5$.

Fig. 7. Microstructures of the AS sample deformed to various strains at 773 K. (a) 0.2 , (b) 0.5 , (c) 0.9 and (d) 1.5 .

Fig. 8. Typical SEM images of the AS samples deformed to various strains at $773 \mathrm{~K}$. (a) $\varepsilon=0.2$, (b) $\varepsilon=0.5$, (c) and (d) $\varepsilon=1.5$.

Fig. 9. TEM micrograph and corresponding diffraction pattern of the AS sample deformed to $\varepsilon=1.5$ at $773 \mathrm{~K}$.

Fig. 10. Variations in the volume fraction (a) and the average grain size (b) of UFG developed with straining at $773 \mathrm{~K}$ for both the AA and AS sample.

Fig. 11. Typical OIM maps and corresponding $\{0001\}$ pole figures of the (a) AA and (b) AS samples deformed to $\varepsilon=1.5$ at $773 \mathrm{~K}$.

Fig. 12. Effect of heat treatment on typical tensile stress-strain curves tested at room temperature for $\mathrm{Mg}-\mathrm{Nd}-\mathrm{Zn}-\mathrm{Zr}$ alloy hot deformed to $\varepsilon=1.5$ at various temperatures from $683 \mathrm{~K}$ to $773 \mathrm{~K}$.

Fig. 13. Changes in tensile mechanical properties at room temperature for $\mathrm{Mg}-\mathrm{Nd}-\mathrm{Zn}-\mathrm{Zr}$ alloy after hot deformation to $\varepsilon=1.5$ with previous compression temperatures. 


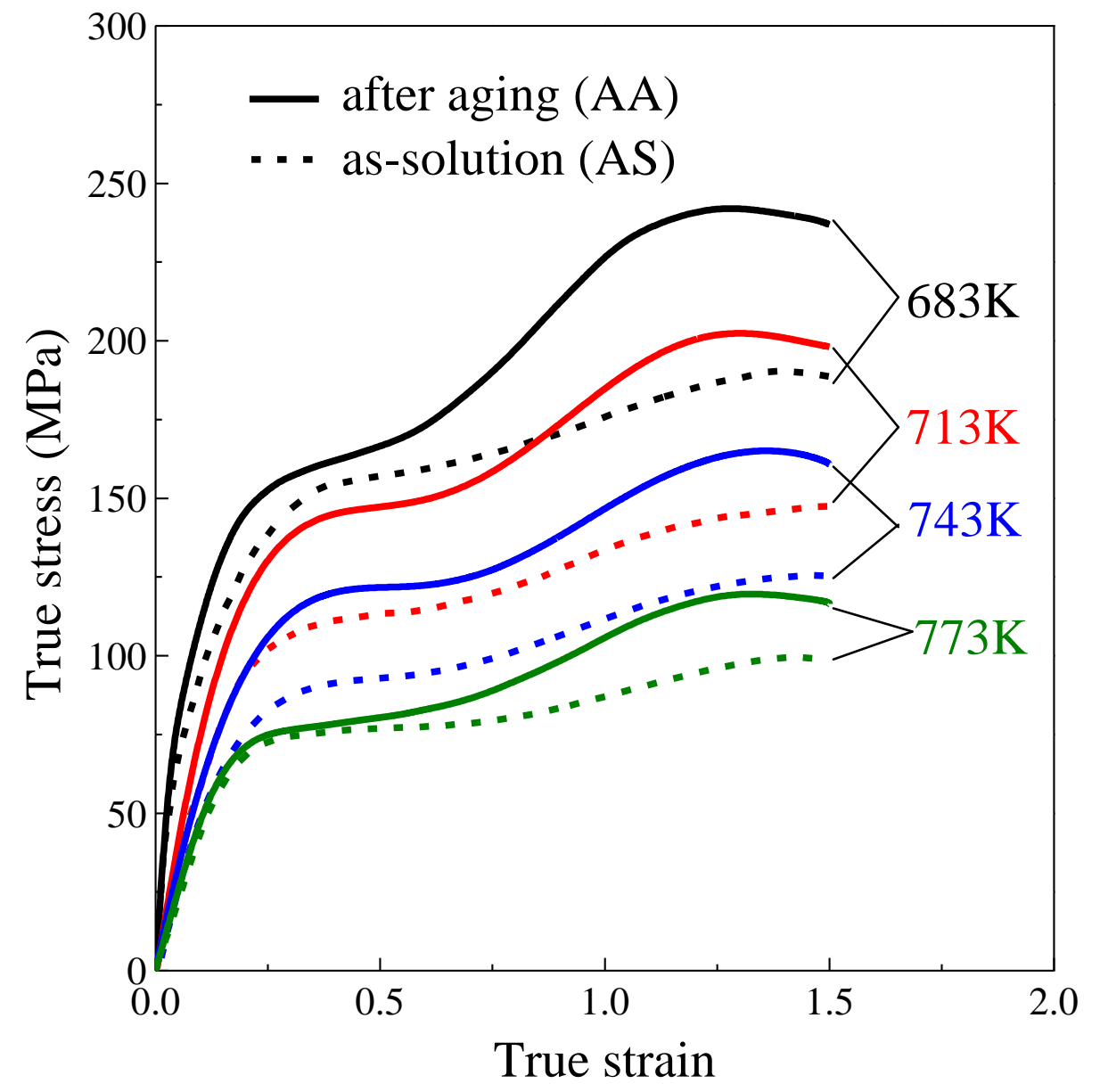

Fig. 1. Temperature dependence of true stress - true strain curves at $\dot{\varepsilon}=0.3 \mathrm{~s}^{-1}$ for $\mathrm{Mg}-\mathrm{Nd}-\mathrm{Zn}-\mathrm{Zr}$ alloy treated in as-solution (broken line) and subsequent aging (solid line). 

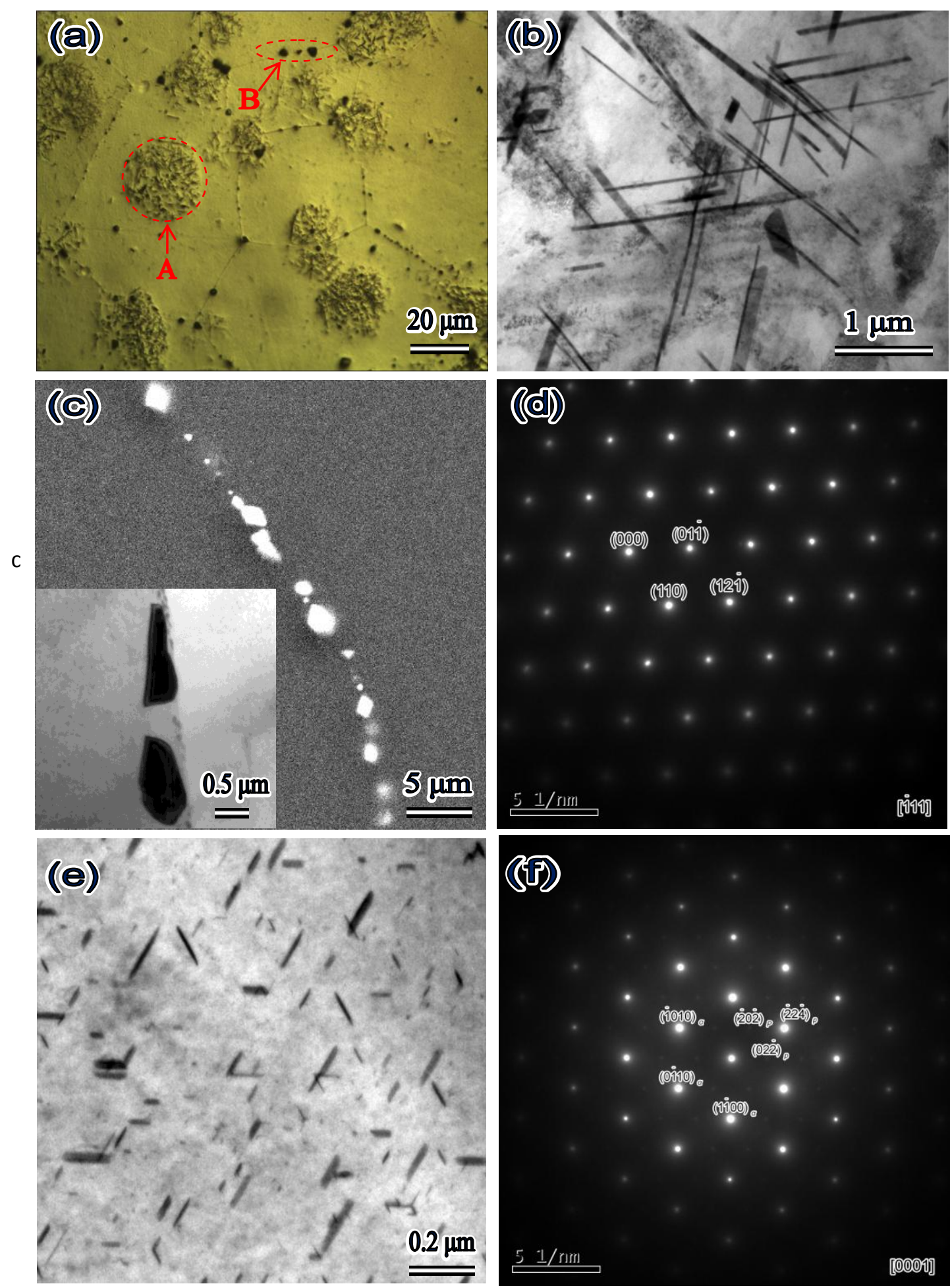

Fig. 2. Microstructures of the AA sample heated at $773 \mathrm{~K}$ for $5 \mathrm{~min}$ before deformation. (a) optical micrograph, (b) TEM micrograph of some clusters of $\mathrm{Zn}-\mathrm{Zr}$ intermetallic phases marked ' $\mathrm{A}$ ' in (a), (c) SEM and TEM and (d) corresponding SADP of the precipitates at grain boundary marked 'B' in (a), (e) TEM and (f) corresponding SADP of the precipitated particles in grain interiors. 

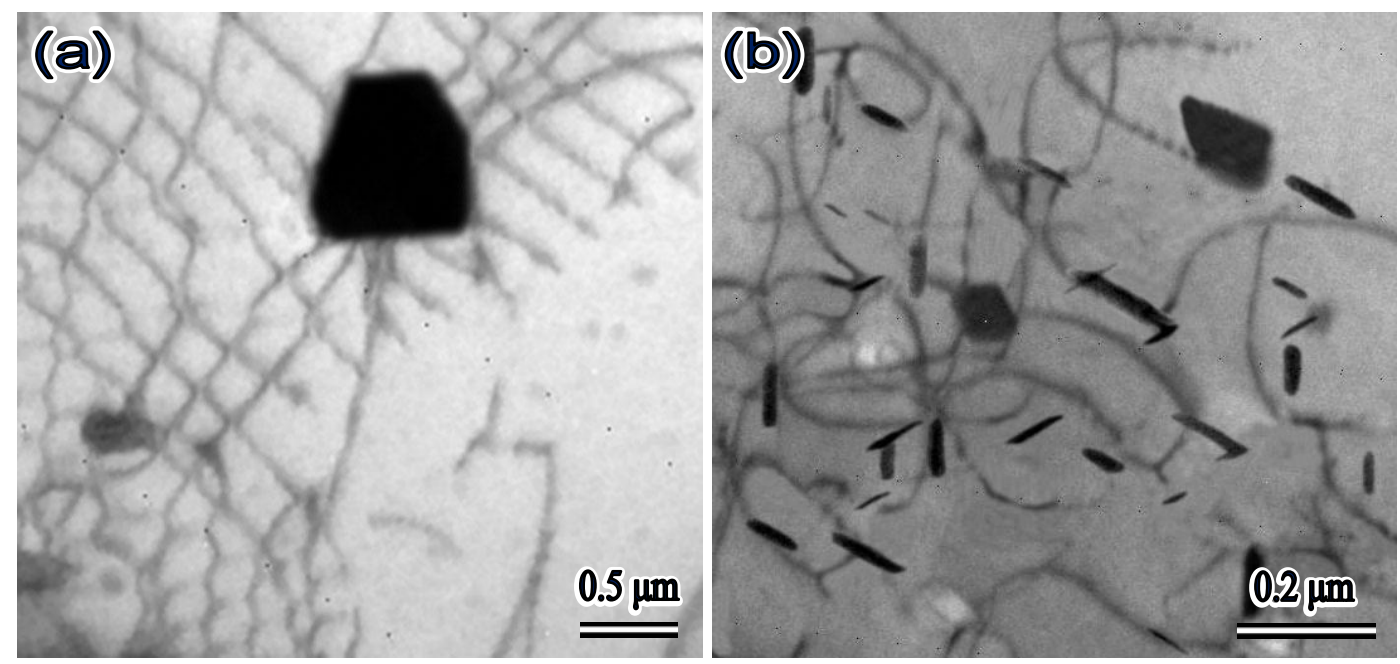

Fig. 3. TEM image of the AA sample deformed to $\varepsilon=0.2$ at $773 \mathrm{~K}$. 


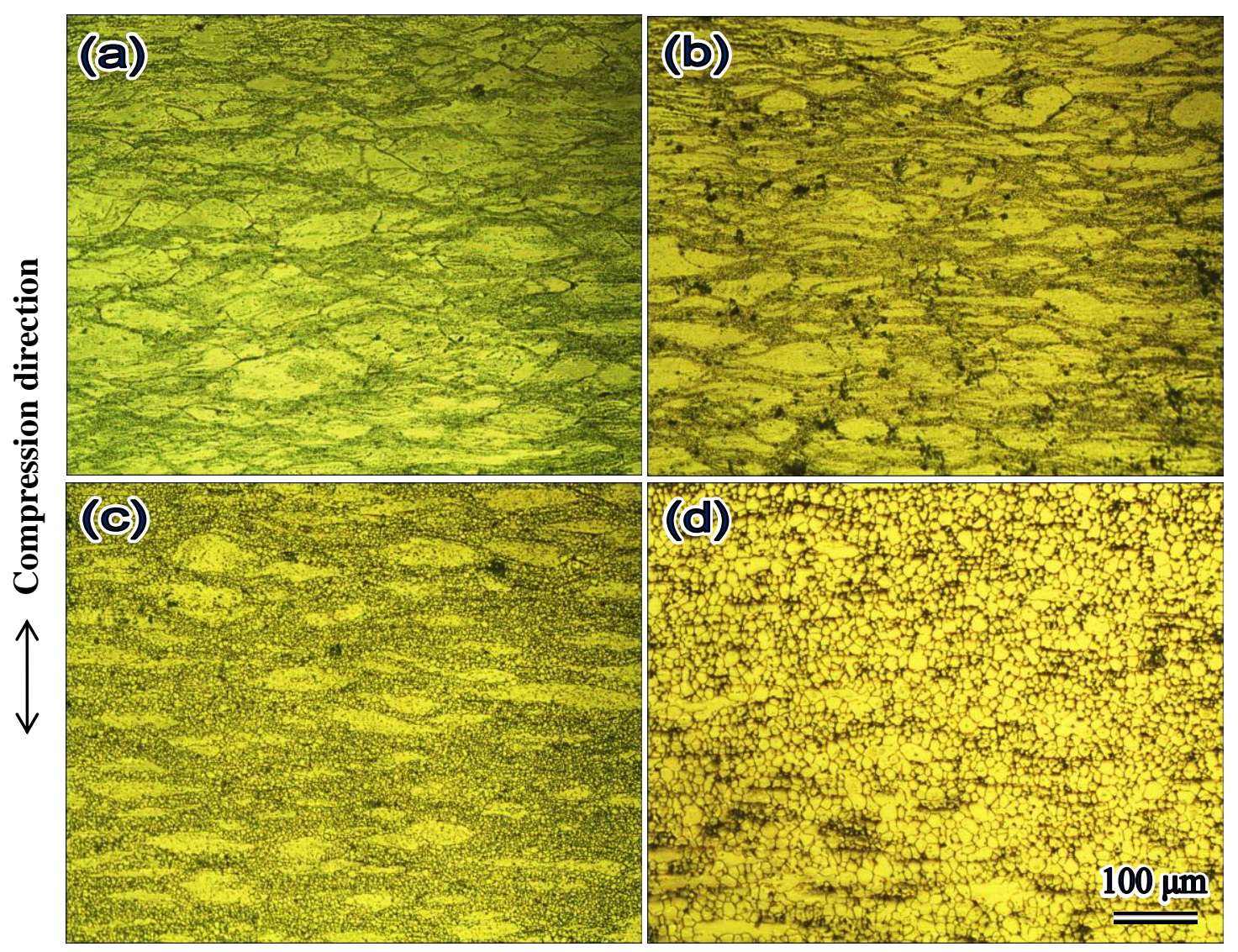

Fig. 4. Microstructures of the AA sample deformed to a strain of 1.5 at various temperatures. (a) $683 \mathrm{~K}$, (b) $713 \mathrm{~K}$, (c) $743 \mathrm{~K}$ and (d) $773 \mathrm{~K}$. 


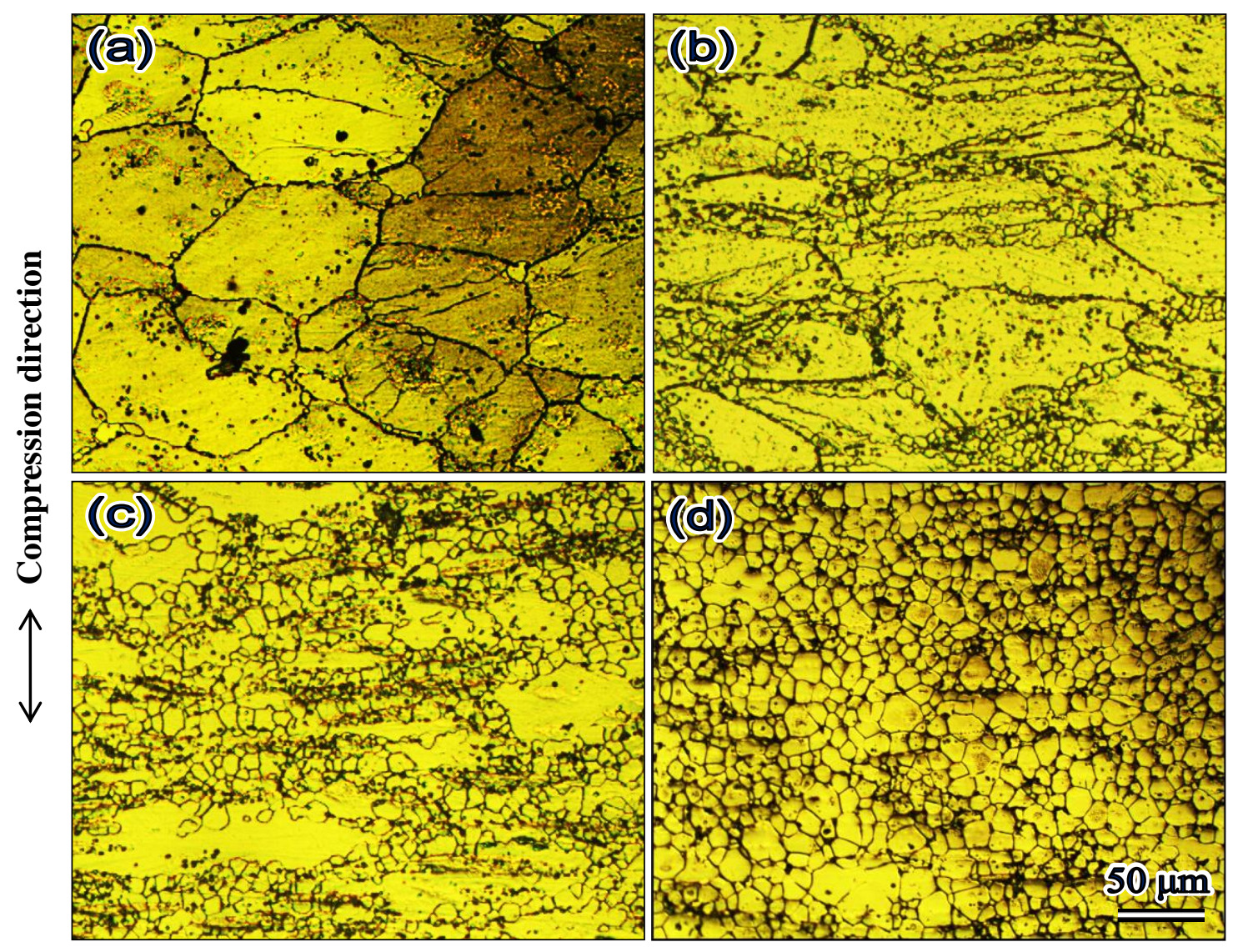

Fig. 5. Microstructures of the AA sample deformed to various strains at 773 K. (a) 0.2 , (b) 0.5 , (c) 0.9 and (d) 1.5. 


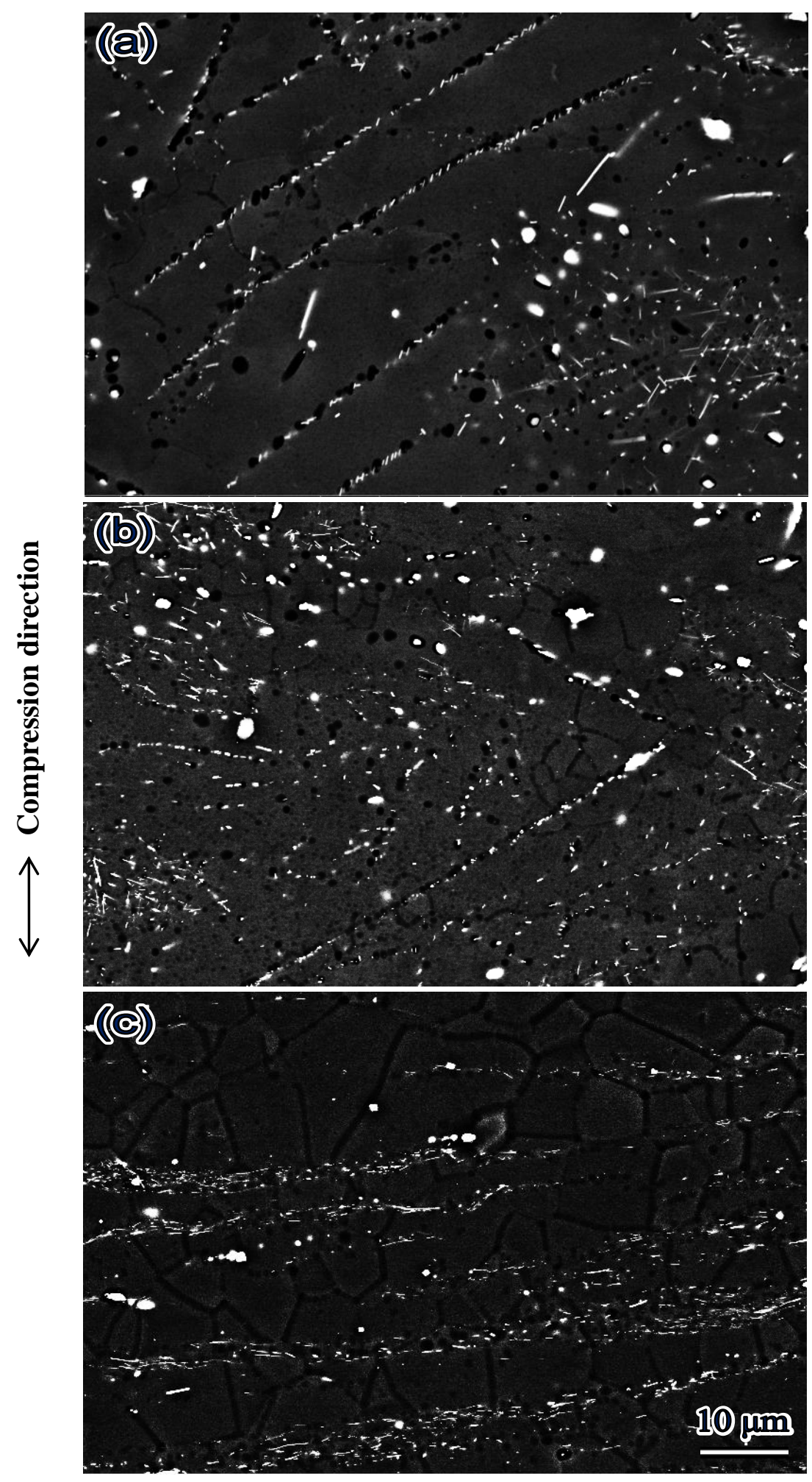

Fig. 6. Typical SEM images of the AA samples deformed to various strains at $773 \mathrm{~K}$. (a) $\varepsilon=0.2$, (b) $\varepsilon=0.5$, (c) $\varepsilon=1.5$. 


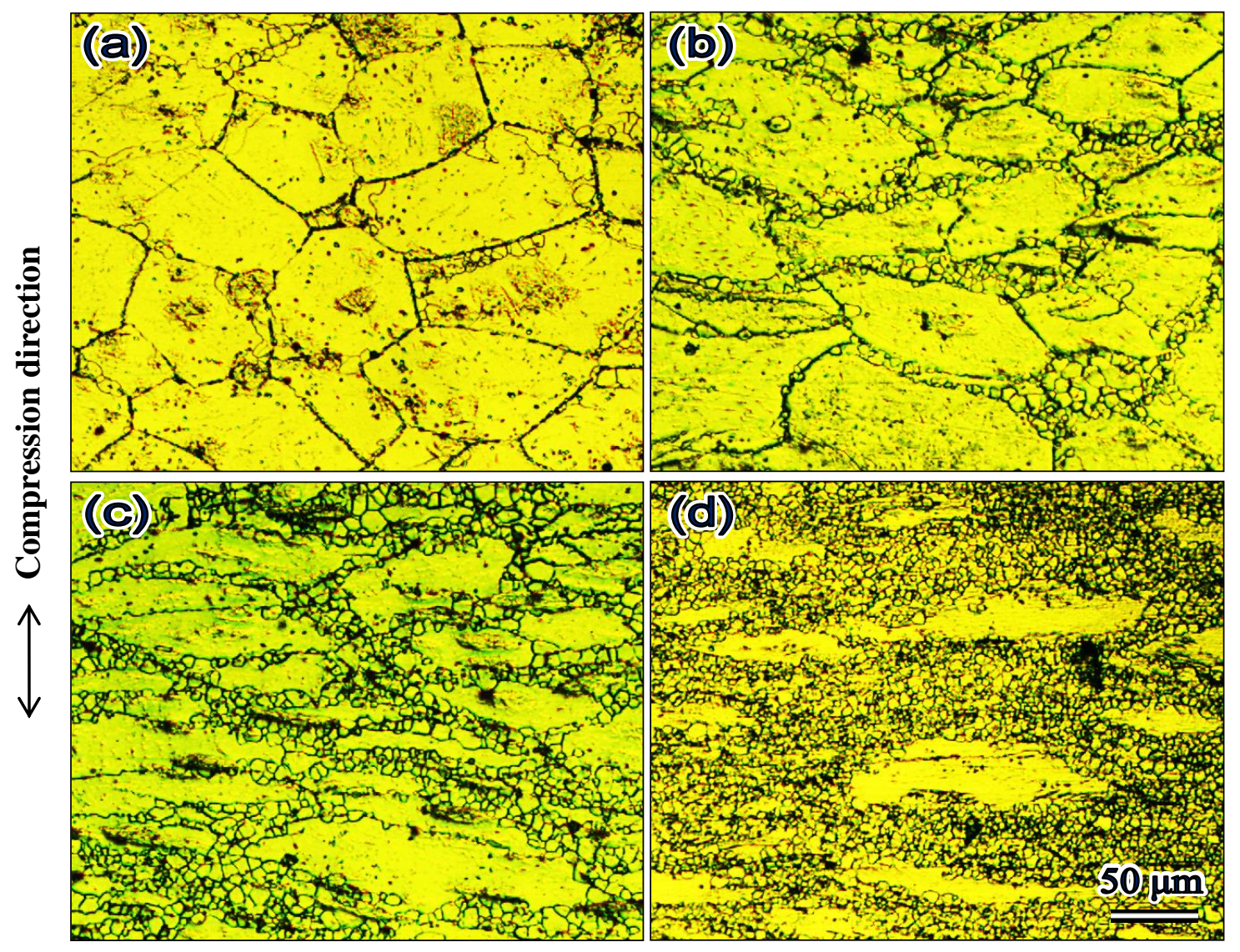

Fig. 7. Microstructures of the AS sample deformed to various strains at 773 K. (a) 0.2 , (b) 0.5 , (c) 0.9 and (d) 1.5. 

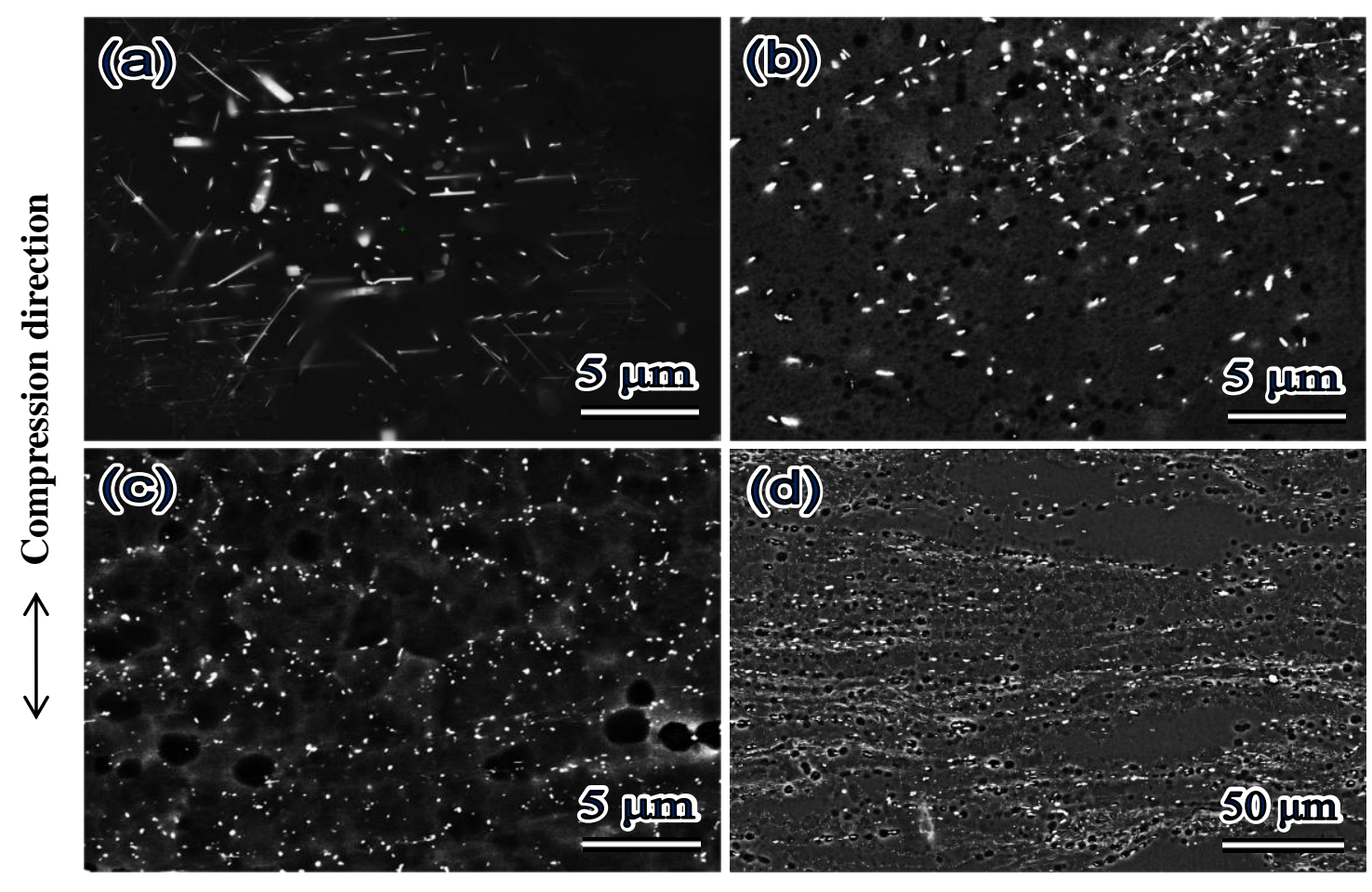

Fig. 8. Typical SEM images of the AS samples deformed to various strains at $773 \mathrm{~K}$. (a) $\varepsilon=0.2$, (b) $\varepsilon=0.5$, (c) and (d) $\varepsilon=1.5$. 


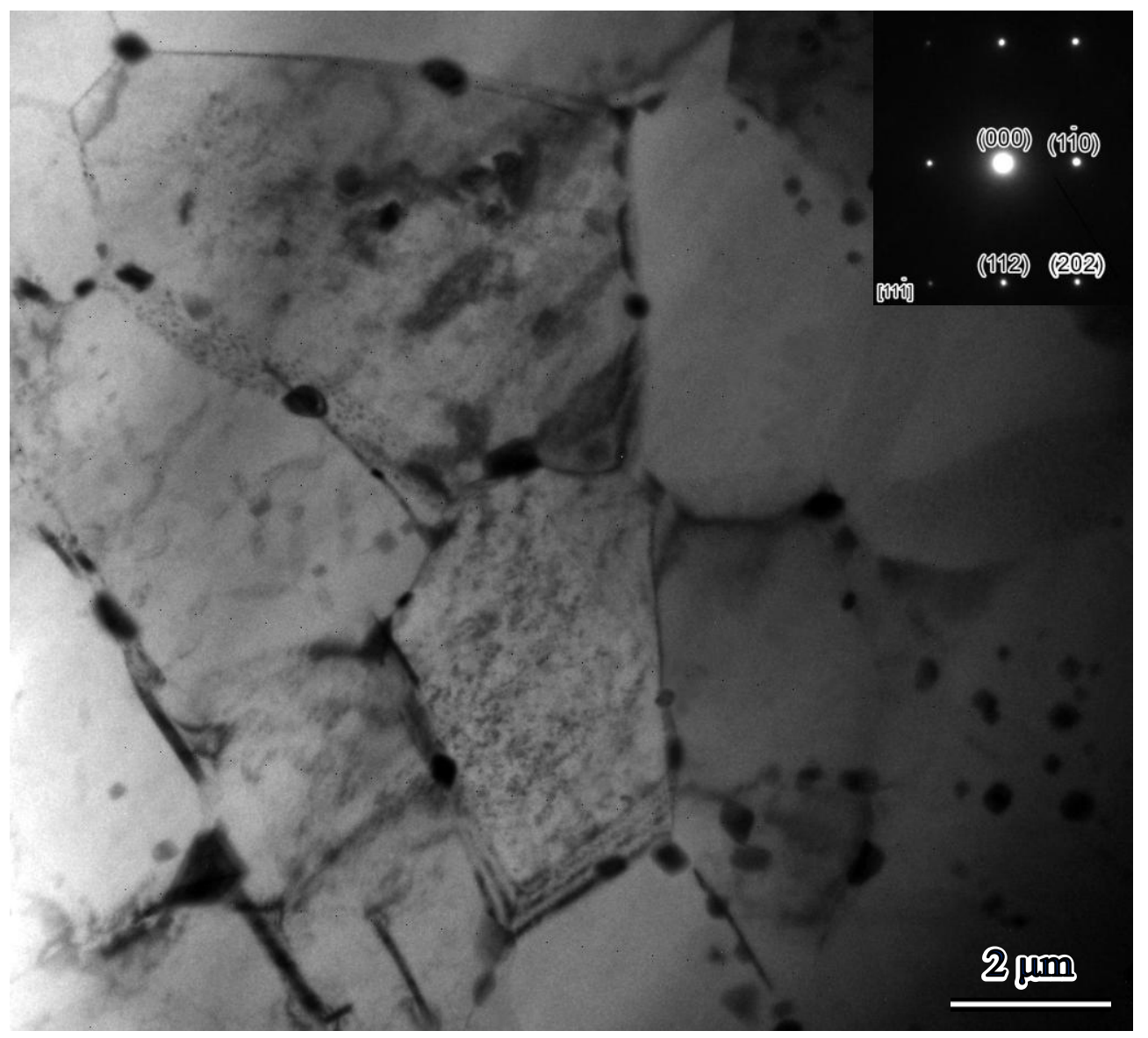

Fig. 9. TEM micrograph and corresponding diffraction pattern of the AS sample deformed to $\varepsilon=1.5$ at $773 \mathrm{~K}$. 


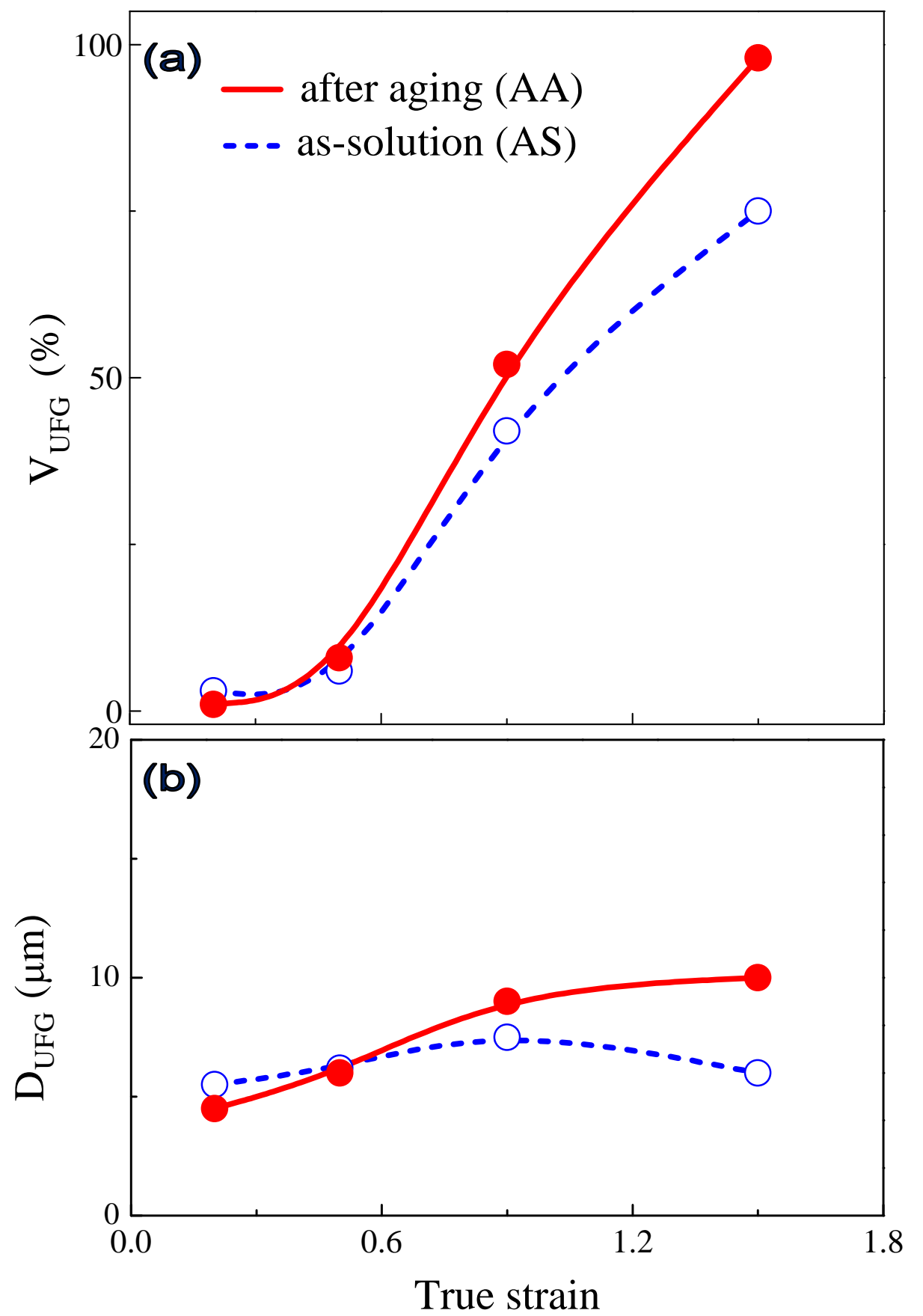

Fig. 10. Variations in the volume fraction (a) and the average grain size (b) of UFGs developed with straining at $773 \mathrm{~K}$ for both the AA and AS sample. 


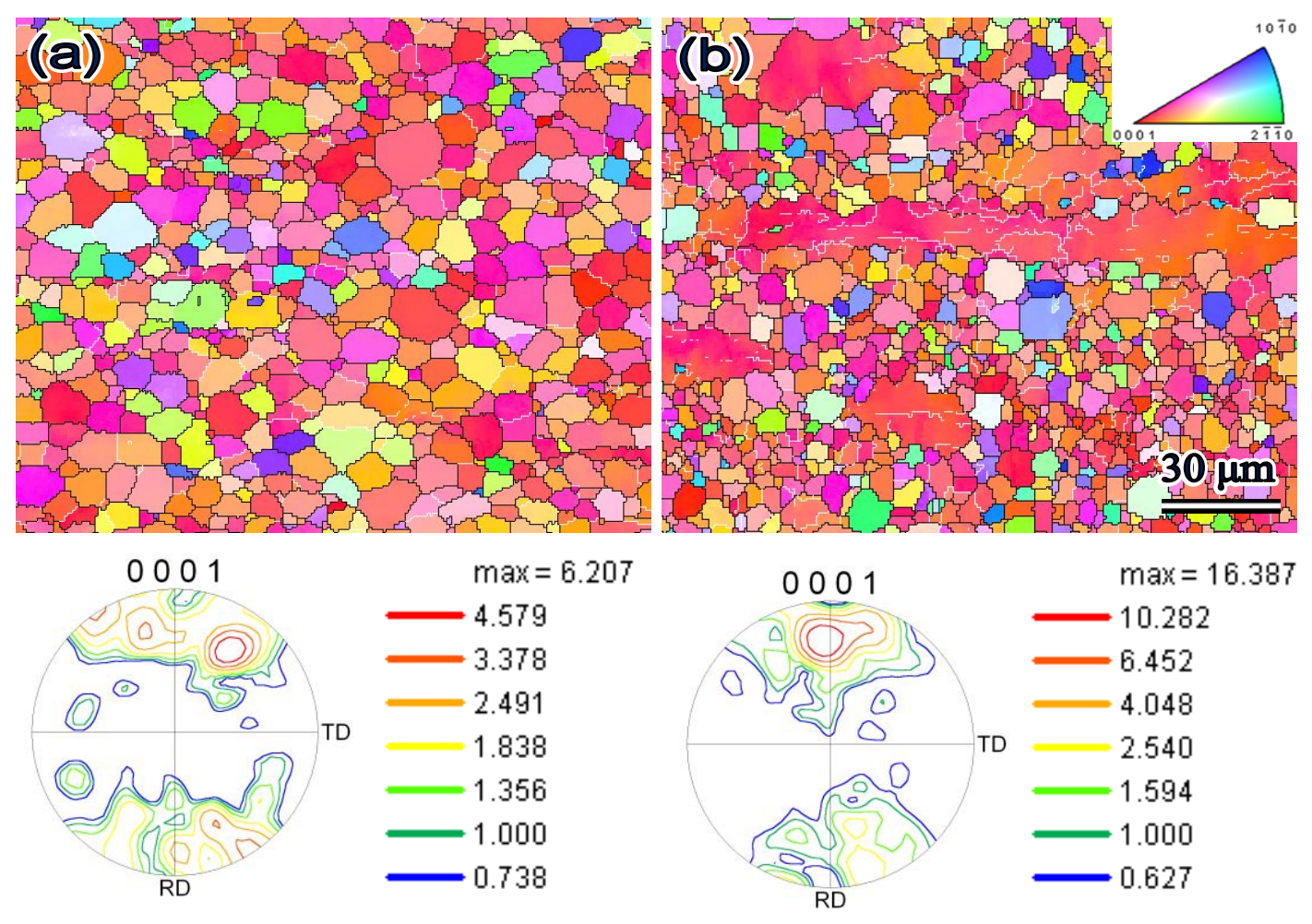

Fig. 11. Typical OIM maps and corresponding $\{0001\}$ pole figures of the (a) AA and (b) AS samples deformed to $\varepsilon=1.5$ at $773 \mathrm{~K}$. 


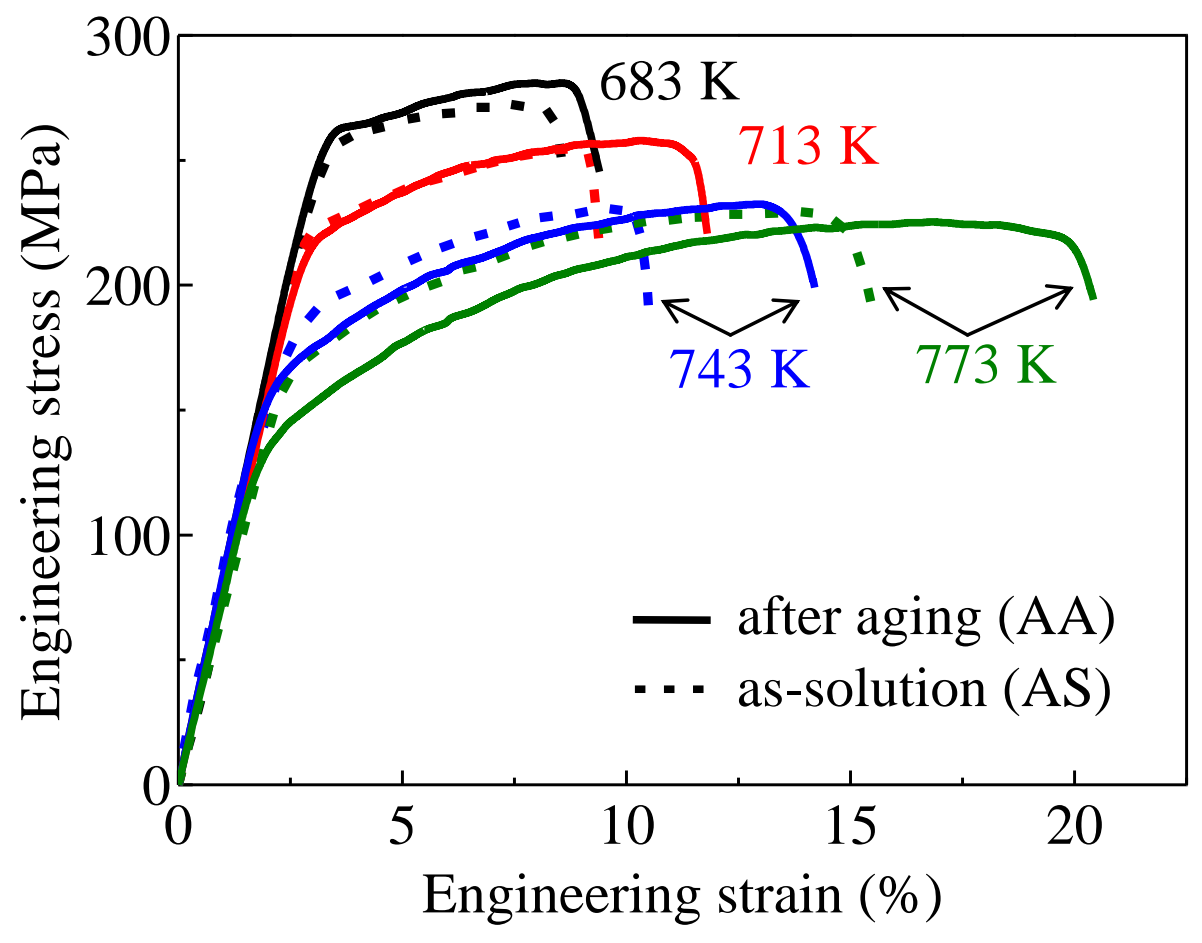

Fig. 12. Effect of heat treatment on typical tensile stress-strain curves tested at room temperature for $\mathrm{Mg}-\mathrm{Nd}-\mathrm{Zn}-\mathrm{Zr}$ alloy hot deformed to $\varepsilon=1.5$ at various temperatures from $683 \mathrm{~K}$ to $773 \mathrm{~K}$. 


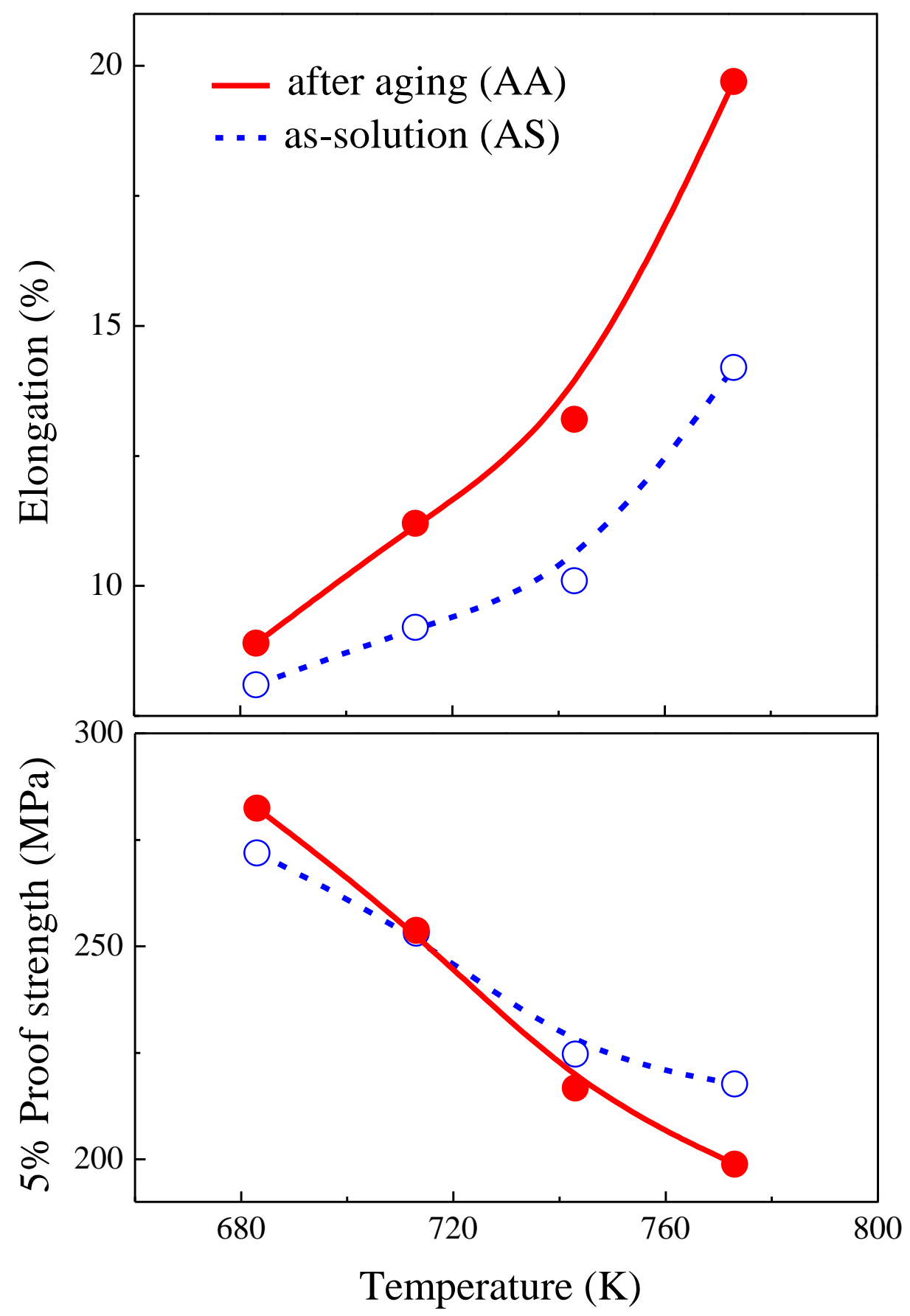

Fig. 13. Changes in tensile mechanical properties at room temperature for $\mathrm{Mg}-\mathrm{Nd}-\mathrm{Zn}-\mathrm{Zr}$ alloy after hot deformation to $\varepsilon=1.5$ with previous compression temperatures. 